\section{AN APPROACH TO THE DIAGNOSIS OF RARE LUNG TUMOURS}

\section{Andrew G. Nicholson}

Department of Histopathology, Royal Brompton and Harefield NHS Foundation Trust and Imperial College School of

Medicine, London, UK

Any primary tumour arising in the lung apart from carcinomas and carcinoids falls under the umbrella term of a 'rare lung tumour'. This therefore includes other epithelial tumours, mesenchymal tumours, lymphomas, and even teratomas and melanomas. Their classification has been updated in the 2015 WHO classification, with the last decade seeing greater understanding of cellular origin, such as sclerosing haemangioma being definitively identified as a low-grade epithelial neoplasm. New tumours with specific genetic abnormalities have also been identified (e.g., pulmonary myxoid sarcoma with EWSR1CREB1 translocation), and lymphangioleiomyomatosis and Langerhans cell histiocytosis are now also classified as neoplasms.

It is useful therefore to have a uniform approach to a potential 'rare' neoplasm. I first apply the 2015 WHO classification, then correlate this with morphological (e.g., clear cell, spindle cell etc.) differential diagnoses and the known immunohistochemical profile to see whether the proposed diagnosis is consistent with these data. I then review the case again in relation to whether the presentation of the tumour is appropriate for my proposed diagnosis (benign/low-grade versus malignant cytology, tumour location, solid/cystic, local/diffuse) and finally review once more in the context of whether tumour might be a secondary process or even a reactive process.

\section{HUNTING FOR THE SIGNATURES OF CANCER BY PLASMA DNA SEQUENCING}

Rossa W.K. Chiu

Li Ka Shing Institute of Health Sciences and Department of Chemical Pathology, Faculty of Medicine, The Chinese University of Hong Kong, Hong Kong Special Administrative Region

Liquid biopsy refers to the analysis of DNA released by tumours into the circulation of patients for the detection, prognostication, selection of therapeutic targets or monitoring of malignancies. For example, regulatory approval has been granted for the detection of epidermal growth factor receptor mutations in plasma of patients with non-small cell lung cancers as a means to stratify therapy. However, the key challenge in the diagnostics of cancer is about its early detection. Given that cancers are highly heterogeneous, our group has been developing whole genome approaches to detect the signatures of cancer via a liquid biopsy. The rationale is to extract as much of the molecular information as possible from a plasma sample to catch a glimpse of the possible presence of cancer. Genomewide approaches to detect tumour-associated copy number aberrations, molecular size profile, DNA methylation signatures and transcriptomic features from circulating nucleic acids have been developed. We have further developed an approach to pinpoint the anatomical location of malignancy, naming to determine a tissue map non-invasively. In summary, many facets of molecular information could be extracted from a liquid biopsy.

Acknowledgement: Supported by the Research Grants Council of the Hong Kong SAR Government under the Theme-based research scheme (T12-404/11).

\section{AN APPROACH TO THE DIAGNOSIS OF INTERSTITIAL PNEUMONIAS IN ADULTS AND CHILDREN}

Andrew G. Nicholson

Department of Histopathology, Royal Brompton and Harefield NHS Foundation Trust and Imperial College School of

Medicine, London, $U K$

In adults with an idiopathic interstitial pneumonia (IIP), an updated classification system was published in 2013. Although definitions for the seven histological patterns remain essentially unchanged, there has been substantial progress in our understanding. In relation to usual interstitial pneumonia (UIP), updated management recommendations were published in 2011, with the most significant change recommending varying degrees of confidence in diagnosis. A pattern of UIP is also being increasingly recognised in patients with chronic hypersensitivity pneumonitis. Idiopathic non-specific interstitial pneumonia is now better defined as a specific clinico-pathological entity, with the majority cases proving to have secondary associations/causes after multidisciplinary review. Respiratory bronchiolitisinterstitial lung disease is now commonly diagnosed without surgical biopsy, and acute exacerbation of IIPs is now well defined. A group of rare entities, including pleuroparenchymal fibroelastosis and rare histological patterns, is also introduced. There have also been advances in the undertaking of biopsies with most institutions sampling at least two sites, ideally using preoperative targeting. These histological patterns are also recognised in children, although classification is more complex and includes entities specific to a younger age group (e.g., neuroendocrine hyperplasia of infancy, pulmonary interstitial glycogenosis). Surfactant protein gene mutations are also being increasingly identified.

\section{B-CELLS BEHAVING BADLY I: A BETTER BASIS TO BEHOLD BELLIGERENCE IN SMALL B-CELL LYMPHOMAS}

Adam Bagg

Department of Pathology and Laboratory Medicine, University of Pennsylvania, Philadelphia, PA, USA

Small B-cell lymphomas and leukaemias (SBCLs) are a clinically, morphologically, immunophenotypically and genetically heterogeneous group of neoplasms that include chronic lymphocytic leukaemia/small lymphocytic lymphoma (CLL/SLL), mantle cell lymphoma (MCL), follicular lymphoma (FL), lymphoplasmacytic lymphoma (LPL), marginal zone lymphoma (MZL) and hairy cell leukaemia (HCL). The pathogenesis of some of these lymphoid malignancies is characterised by distinct translocations, for example $\mathrm{t}(11 ; 14)$ and $\mathrm{t}(14 ; 18)$, respectively, in most cases of MCL and FL. By contrast, other entities are associated with a variety of non-specific and non-diagnostic but nevertheless recurrent and prognostically pertinent numeric 
abnormalities, such as del(17p), del(13q14), del(11q22) and +12 in CLL. Yet others like LPL and HCL lack specific cytogenetic aberrations. The recent surge in next generation sequencing technology has shed abundant light on the genetic landscape of SBCLs through characterisation of numerous driver mutations including BRAF (V600E) in HCL and MYD88 (L265P) in LPL, amongst others. These significant advances and panoply of available tests notwithstanding, it is important to appreciate that not all SBCLs need genetic testing for diagnosis, not all harbour their characteristic genetic features and not all are diagnostically specific. This lecture will attempt to review the role of genetic studies in SBCLs, highlighting their advantages and limitations.

\section{B-CELLS BEHAVING BADLY II: A BETTER BASIS TO BEHOLD BELLIGERENCE IN AGGRESSIVE B-CELL LYMPHOMAS}

\author{
Adam Bagg \\ Department of Pathology and Laboratory Medicine, University \\ of Pennsylvania, Philadelphia, PA, USA
}

Aggressive B-cell lymphomas include diffuse large B-cell lymphoma (DLBCL), the most common type of lymphoma encountered in most countries. Despite the single term, DLBCLs are a rather diverse group of lymphomas that can be subclassified using a variety of different approaches, including anatomical site, morphology, immunophenotype, and a number of genetic strategies. Nascent molecular technologies have led to the discovery of many of the genetic events underlying the pathogenesis of this group of diseases. Whether by defining gene signatures that subclassify DLBCL into subgroups based upon cell-of-origin, through dysregulation of key cellular pathways, or specific mutations, we are approaching an era in which personalised diagnostics, prognostication and therapy are imminent. Attempts to develop surrogate immunohistochemical algorithms for some of these genetic phenomena have been variably successful. This lecture will focus on DLBCL and highlight one particular shade of so-called grey zone lymphoma and its relationship to double-hit lymphomas. An attempt will also be made to synthesise the rational use of genetic testing of lymphomas covered in both this and the preceding lecture on small B-cell neoplasms.

\section{SYSTEMIC AND VASCULAR DISEASES IN THE LUNGS}

\section{Andrew G. Nicholson}

Department of Histopathology, Royal Brompton and Harefield NHS Foundation Trust and Imperial College School of Medicine, London, UK

Although many patients with systemic and vascular diseases involving the lungs are diagnosed without recourse to biopsy, pathologists will still be involved on occasion. Indeed, it is often only after multidisciplinary review that the possibility of a systemic cause for the pulmonary symptoms is considered. Clinical correlation is therefore essential, also in relation to the possibility of iatrogenic disease (e.g., drug reactions). In relation to microscopy, for connective tissue disorders in particular, it is important to remember that these diseases can affect all compartments of the lung. As an example, rheumatoid disease is not only associated with patterns of interstitial pneumonia, but also, rheumatoid nodules, apical fibrosis, airways disease (follicular bronchitis, bronchiectasis, bronchocentric granulomatosis, constrictive obliterative bronchiolitis), pulmonary hypertension, and rarely development of malignancies in the lung. Also, it is important to remember that one pattern of disease may progress to another, dependent on the disease process, so longitudinal behaviour is an additional key factor when reviewing samples. In relation to pulmonary vascular disease, excluding vascular tumours, there also remains a minority of cases where biopsy still plays a role (e.g., veno-occlusive disease, embolic disease). Typically, a surgical lung biopsy is required, although transbronchial biopsy may rarely yield information.

\section{CUTANEOUS MANIFESTATIONS OF SYSTEMIC DISEASE}

Rooshdiya Z. Karim $^{1,2}$

${ }^{1}$ Department of Tissue Pathology and Diagnostic Oncology, Royal Prince Alfred Hospital, Sydney, and ${ }^{2}$ University of Sydney, Faculty of Medicine, Central Clinical School, Sydney, NSW, Australia

The cutaneous manifestations of systemic diseases are vast and varied. The skin is our largest organ and can act as a mirror to reflect internal pathology including infections; immune, autoimmune and rheumatic diseases; genetic, metabolic, endocrine and nutritional states; internal malignancies; cancer syndromes; haematological diseases; and drug effects.

The cutaneous manifestations may provide the prompt to search for the underlying systemic disease, may assist in classification of the systemic disease and can be the main symptoms of distress for the patient; hence, accurate pathological diagnosis with clinical correlation is critical.

\section{WHAT DO I NEED TO KNOW ABOUT SOMATIC MUTATION TESTING AS A TISSUE PATHOLOGIST - A SURVIVAL GUIDE?}

Sandra O'Toole

Molecular Diagnostic Oncology, Department of Tissue Pathology and Diagnostic Oncology, Royal Prince Alfred Hospital, and University of Sydney, Sydney, NSW, Australia

New technologies have enabled high throughput and detailed analysis of the genetic changes in a range of tumours with increasing recognition that these may assist in the diagnosis and selection of treatment.

In subsets of patients with advanced non-small cell lung cancer, melanoma and colorectal carcinoma, genetic analysis and matched targeted therapies have become standard of care. In lymphomas, detection of MYC gene rearrangement is increasingly used to assist in treatment selection. Characteristic gene rearrangements have been identified in up to 53 different bone and soft tissue tumours as well as in a range of head and neck and renal tumours among others.

Tissue pathologists need to develop a working understanding of the principles of techniques, their advantages and disadvantages, and critically, how to integrate molecular results into the diagnostic process. This lecture will outline principles of molecular assays and technologies, the key indications for their use, and 